\title{
A Study to Evaluate Use of Antibiotics in Sore Throat to Eliminate Their Irrational Use, Using Centor Criteria and ASO Titre
}

\author{
Ramesh Kumar Saidugari ${ }^{1}$, Lingaiah Jadi², Deepak Kumar Phulwani ${ }^{3}$, Vineel Muppidi ${ }^{4}$, Sachin Gurnule ${ }^{5}$ \\ ${ }^{1}$ Department of ENT, Chalmeda Anand Rao Institute of Medical Sciences, Bommakal, Karimnagar, Telangana, India. \\ 2Department of ENT, Chalmeda Anand Rao Institute of Medical Sciences, Bommakal, Karimnagar, Telangana, India. \\ ${ }^{3}$ Department of ENT, Chalmeda Anand Rao Institute of Medical Sciences, Bommakal, Karimnagar, Telangana, India. \\ ${ }^{4}$ Department of ENT, Chalmeda Anand Rao Institute of Medical Sciences, Bommakal, Karimnagar, Telangana, India. \\ ${ }_{5}^{5}$ epartment of ENT, Chalmeda Anand Rao Institute of Medical Sciences, Bommakal, Karimnagar, Telangana, India.
}

\section{ABSTRACT}

\section{BACKGROUND}

Use of antibiotics in sore throat is now getting out of hand as it has become very common to prescribe antibiotics for every sore throat patient. This has now led to increased antibiotic resistance in India. We wanted to evaluate proper use of antibiotics in cases of sore throat.

\section{METHODS}

A prospective study was done in 261 patients who were clinically diagnosed as acute tonsillitis or acute pharyngitis. Study was undertaken in a set up where most of the patients are from rural areas. Patients were analysed using Centor criteria and ASO (anti-streptolysin 0 where 0 stands for oxygen labile) titre for confirmation of streptococcal infection, and for negative results throat culture was done and antibiotics were prescribed accordingly and data was analysed by using chi square test for qualitative data with the help of statistical software SPSS v.25, and MS Excel. A p value of $<0.05$ was considered as statistically significant.

\section{RESULTS}

Results showed that $26.05 \%$ patients needed antibiotics while the rest $74 \%$ did well without antibiotics.

\section{CONCLUSIONS}

Centor criteria can be used to determine as to which patients need antibiotics straight away and ASO titer can be a useful test for poor patients as it can help in determining who actually needs antibiotics thus eliminating the irrational use.

\section{KEY WORDS}

ASO Titre, Beta Haemolytic Group A Streptococcus, Antibiotic Resistance, Centor Criteria, RAST, Sore Throat, Tonsillitis, Pharyngitis
Corresponding Author:

Dr. Lingaiah Jadi,

Professor,

Department of ENT,

Chalmeda Anand Rao Institute of Medical Sciences, Bommakal, Karimnagar-505001,

Telangana, India.

E-mail:drjadi_ms@yahoo.com

DOI: $10.14260 / j e m d s / 2019 / 796$

Financial or Other Competing Interests: None.

How to Cite This Article:

Saidugari RK, Jadi L, Phulwani DK, et al. A study to evaluate use of antibiotics in sore throat to eliminate their irrational use, using centor criteria and ASO titre. J. Evolution Med. Dent. Sci. 2019;8(49): 3679-3682, DOI:
Submission 16-09-2019,

Peer Review 17-11-2019,

Acceptance 23-11-2019,

Published 09-12-2019. 


\section{BACKGROUND}

Sore throat (Acute tonsillitis/acute pharyngitis) is one of the most common entity that is encountered in an ENT Outpatient Department. Viruses are responsible for most of the cases but a few sore throat cases are caused by Group A Streptococcus (GAS) ( $\beta$ haemolytic/ $\beta \mathrm{HSA}$ ) as well. ${ }^{1}$ So it becomes extremely important to confirm the causative organism before prescribing antibiotics as there is a concern regarding the increasing resistance for antibiotics in clinical practice and their side effects. ${ }^{2,3}$ To avoid irrational usage one can use Centor criteria with any of these tests like ASO titre, anti-DNAse B, RAST(Rapid antigen streptococcus tests), Throat swab culture and then antibiotics can be started accordingly. This would help not only to curb the incautious use of antibiotics but also avoiding its adverse effects. A study conducted on 50 patients showed that ASO titre alone cannot be reliably used as a criteria to perform tonsillectomy. ${ }^{4}$ To diagnose group A streptococcal pharyngitis among adults RAST immunochromatographic methods seems to be very sensitive and highly specific. ${ }^{5}$ It has become well recognized that microorganisms including bacteria, fungi, parasites, and viruses are capable of developing resistance by reckless use of antibiotics and now there's the threat of Antimicrobial Resistance on the Human Microbiome. ${ }^{6}$

The present study was undertaken in a rural area where people have minimum to spend. So, a tool like Centor criteria helps to put them into separate groups, like patients who score $=>4$ are at a higher risk of $\beta$ haemolytic GAS infection and the one with low score in Centor criteria pointing towards a viral infection. ASO titre was done instead of RAST because ASO titre being a cheaper test lures poor people to get the test done, and also it provides an idea about $\beta$ haemolytic GAS infection thus a cheaper tool than RAST that can be used in rural INDIA. While using ASO titre we followed our exclusion criteria strictly to eliminate as many as false positives as possible. But if the patient can afford RAST will always be the gold standard for rapid antigen detection on OPD basis. We wanted to evaluate the use of antibiotics in sore throat patients.

\section{METHODS}

A prospective study of 261 patients was carried out between January 2018 to December 2018. A predesigned and selfadministered questioner was used to collect the relevant data (Patient's data, clinical findings, investigation reports) from the individual patient selected according to inclusion criteria and exclusion criteria. Age group of patients was between 5 to 60 years. Centor ${ }^{7}$ criteria was used to analyse the patients.

ASO (Anti-streptolysin 0 where 0 stands for oxygen labile) titre was done on patients and the results were analysed. If ASO was positive patients were prescribed Coamoxiclav (Or clarithromycin to penicillin allergic patients). If the results were negative patients were treated symptomatically with pain killers and throat swab was taken and sent for culture and sensitivity. Follow up was done at 7 days and Centor criteria was again used to assess the patients. Absence of fever, resolution of anterior cervical adenitis and tonsillar exudate was considered as relieved.
Any patient that fulfilled the inclusion criteria was evaluated on the following scale according to Centor criteria (1 point for each of following was given)

\section{Inclusion Criteria}

- Temperature $>101.3^{\circ} \mathrm{F}$.

- Membranous/ follicular tonsillitis/ pharyngitis.

- Anterior cervical adenitis.

- Negative for cough.

- Age: less than 15 years. (subtract 1 if age more than 45 years).

\section{Exclusion Criteria}

- Patients on or already received antibiotics in previous weeks.

- Known cases of rheumatic fever.

- Cardiac valvular lesions cases.

- Patients with diabetes, tuberculosis, malignancies, renal failure, immunocompromised status.

- Patients with any liver disease (where the presence of high lipoprotein concentrations in the serum may mimic antibody activity).

\section{Ethical Consideration}

The study was conducted after getting ethical approval from ethical committee of Chalmeda Anand Rao Institute of Medical Science, Karimnagar, Telangana State, India, and a written consent was taken from all patients to be included in the study.

\section{Statistical Analysis}

The data was analysed by using Descriptive (percentage, proportion) and inferential statistics. (chi-square test for qualitative data) with the help of statistical software SPSS v.25, MS EXCEL. The $p$ value, $<0.05$ considered as statistically significant.

\section{RESULTS}

Of total 261 patients, age distribution is given in Table 1. There were 91 (35\%) males and $170(65 \%)$ females. Out of these 261 patients $42(16.09 \%)$ had Centor criteria of four or more who were prescribed antibiotics and supportive measures straight away. ASO titre was done for all 261 patients and was found to be positive in $54(20.68 \%)$ patients who were prescribed antibiotics. For remaining patients, throat swab culture was done and prescribed an antibiotic only if the culture was positive. $14(5.36 \%)$ patients out of the remaining (207) were found to be culture positive and result informed to patients and they were started on antibiotics.

\begin{tabular}{|c|c|c|}
\hline Age & No. & \% \\
\hline $05-14$ & 44 & 16.85 \\
\hline $15-24$ & 82 & 31.41 \\
\hline $25-34$ & 75 & 28.73 \\
\hline $35-44$ & 26 & 09.96 \\
\hline 45 and above & 34 & 13.02 \\
\hline Total & $\mathbf{2 6 1}$ & $\mathbf{1 0 0 . 0} \%$ \\
\hline \multicolumn{3}{|c|}{ Table 1. Age Distribution of Patients } \\
\hline
\end{tabular}




\begin{tabular}{|c|c|c|}
\hline Centor Criteria & No & $\mathbf{0}$ \\
\hline Centor criteria $\geq 4$ & 42 & 16.09 \\
\hline ASO +ve & 54 & 20.68 \\
\hline ASO -ve, Culture + Ve & 14 & 05.36 \\
\hline ASO -ve, Culture - Ve & 193 & 73.94 \\
\hline Table 2. Patients' Distribution as per Centor Criteria and ASO Titre \\
\hline
\end{tabular}

\begin{tabular}{|c|c|c|}
\hline Antibiotic & No. & \% \\
\hline Prescribed & 68 & 26.05 \\
\hline Not Prescribed & 193 & 73.94 \\
\hline Total & $\mathbf{2 6 1}$ & $\mathbf{1 0 0 . 0} \%$ \\
\hline \multicolumn{2}{|c|}{ Table 3. Antibiotics Prescribed } \\
\hline
\end{tabular}

\begin{tabular}{|c|c|c|c|}
\hline Group & Relieved & Non-Relieved & p-Value \\
\hline Antibiotic & $57(21.8)$ & $11(4.2)$ & \multirow{3}{*}{$0.007^{* *}(\mathrm{Sig})$} \\
\hline Non-Antibiotic & $182(69.7)$ & $11(4.2)$ & \\
\hline Total & $239(91.6)$ & $22(8.4)$ & \\
\hline \multicolumn{4}{|c|}{ Table 4. Relief in Symptoms after Treatment } \\
\hline $\mathrm{p}<0.01$, highly $s$ & int at $5 \%$ leve & ignificance & \\
\hline
\end{tabular}

\section{DISCUSSION}

One of the most common human pathogens causing problem in humans is $\beta$ hemolytic GAS ( $S$. pyogenes). It leads to disease in 3 ways.

- Pyogenic inflammation, induced by organism locally at tissue site,

- Exotoxin production, which leads to systemic invasive disease.

- Immunologic, antibodies against organism show cross reactivity with normal tissue, immune complexes are produced that damage these tissues.

Five important toxins and hemolysis are produced by Group A organisms. Streptolysin O (oxygen labile) is among them and has antigenicity. ASO antibodies develop after Group A Streptococcal infection. Infection with GABHS (group A beta haemolytic streptococci) in any part of the body leads to increase in the serum ASO titre. ${ }^{8}$ Usually a positive ASO titre test is $>200$ units $/ \mathrm{ml}$, but the ASO titre normal value ranges vary between laboratory to laboratory and also by the age of the patient. Age above 5 years ASO titre less than 200 units $/ \mathrm{ml}$ and below the age of 5 years it is less than 100 units $/ \mathrm{ml}$ are considered as normal. The false negatives rate is high. So, a better test is RST. A rapid test for identification of GAS, rapid antigen streptococcus tests (RAST) ${ }^{9}$ can also be done. There are three major types of RST- latex fixation test, optical immunoassay and lateral flow test most widely used RST.

This is a very simple test which can be performed as a hassle-free outpatient procedure. The patient's throat is first swabbed, and sample is collected. The swab has to be rubbed or wiped properly over the tonsils/pharynx and a little pressure over anterior pillar of tonsil can be applied by tongue depressor to take the exudate which can provide best result. In RSTs, the sample collected is then put into a reagent containing antibodies that will bind specifically to a GAS antigen (Lateral flow test). Test is 95\% sensitive and 98\% specific as claimed by manufacturer. 7 to 8 minutes is the approximate time taken by the test.

There are guidelines that recommend either rapid antigen streptococci testing (RAST) or empiric treatment of patients who are more susceptible to have GAS pharyngitis, with neither testing nor treatment for patients very unlikely to have GAS infection ${ }^{10 .}$ Some guidelines tell to limit antibiotic use, and have adapted to do RAST and result should show a positive rapid antigen strep test prior to prescribing antibiotics. ${ }^{11}$ Antibiotics use in clinical practice is incautious and this has led to higher prevalence of multi-drug resistance in India. ${ }^{12}$

An easy to follow criteria is the America association of family physicians. ${ }^{11}$ Centor criteria is first used to analyse patients, if score is $>=4$, were prescribed antibiotic stat as these patients were at higher risk of GAS. Remaining were sent for ASO titre, positive ASO patients were started on antibiotics and negative were started on supportive therapy and throat swab culture was sent for them. Culture positive were prescribed antibiotics while culture negative were just kept on supportive treatment. In this study only $26.05 \%$ patients required antibiotics and rest did well without any antibiotics thus limiting the irrational use of antibiotics. In our study the $p$ value is 0.007 . The $p$ value $<0.05$ considered as statistically significant.

\section{CONCLUSIONS}

Irrational use of antibiotics in cases of tonsillitis/pharyngitis should be a cause of concern and should only be prescribed if bacterial cause is evident. Prescribing unnecessary antibiotics is increasing drug resistance not only in India but globally. This study shows that around 70 to $74 \%$ patients got relieved without using any antibiotics. Clinicians should do all the proper tests like ASO titre or RAST and also follow criteria like Centor criteria to differentiate patients into separate groups before prescribing antibiotics and put a stop to its irrational use.

\section{REFERENCES}

[1] Wolford RW, Schaefer TJ. Pharyngitis. In: StatPearls. Treasure Island (FL): StatPearls Publishing: 2019. [Updated 2019 Feb 28].

[2] Bell BG, Schellevis F, Stobberingh E, et al. A systematic review and meta-analysis of the effects of antibiotic consumption on antibiotic resistance. BMC Infect Dis 2014;14:13.

[3] Faden H, Callanan V, Pizzuto M, et al. The ubiquity of asymptomatic respiratory viral infections in the tonsils and adenoids of children and their impact on airway obstruction. Int J Pediatr Otorhinolaryngol 2016;90:12832.

[4] Hembrom R, Roychaudhuri BK, Saha AK, et al. Evaluation of the validity of high serum antistreptolysin o titre only, as an indication for tonsillectomy. Indian J Otolaryngol Head Neck Surg 2014;66(3):232-6.

[5] Brinkac L, Voorhies A, Gomez A, et al. The threat of antimicrobial resistance on the human microbiome. Microb Ecol 2017;74(4):1001-8. 
[6] Stewart EH, Davis B, Clemans-Taylor BL, et al. Rapid antigen group A streptococcus test to diagnose pharyngitis: a systematic review and meta-analysis. PLoS One 2014;9(11):e111727.

[7] Centor RM, Witherspoon JM, Dalton HP, et al. The diagnosis of strep throat in adults in the emergency room. Med Decis Making 1981;1(3):239-46.

[8] Ramirez A, Peidrola D, Lopez A, et al. Beta-hemolytic streptococci in tonsil hypertrophy and recurrent tonsillitis. Enferm Infecc Microbiol Clin 1997;15(6):3158.

[9] Gerber MA. Comparison of throat cultures and rapid strep tests for diagnosis of streptococcal pharyngitis. Pediatr Infect Dis J 1989;8(11):820-4.
[10] Matthys J, De Meyere M, van Driel ML, et al. Differences among international pharyngitis guidelines: not just academic. Ann Fam Med 2007;5(5):436-43.

[11] Shulman ST, Bisno AL, Clegg HW, et al. Clinical practice guideline for the diagnosis and management of group A streptococcal pharyngitis: 2012 update by the Infectious Diseases Society of America. Clin Infect Dis 2012;55(10):1279-82.

[12] Kumar SG, Adithan C, Harish BN, et al. Antimicrobial resistance in India: a review. Journal of Natural Science, Biology and Medicine 2013;4(2):286-91. 